\title{
Congenital Anomalies: Impact of Prenatal Diagnosis on Mode of Delivery
}

\begin{abstract}
An important aspect of prenatal diagnosis is the avoidance of emergency caesarean delivery (CD) where the abnormality is considered lethal and the infant will not survive. A consecutive cohort of 211,163 women delivered of infants weighing 500 grams or more in three tertiary referral
centers from $01 / 95$ to $12 / 04$, was analyzed for perinatal death attributed to congenital malformations In the group that died in the neonatal centers from $01 / 95$ to $12 / 04$, was analyzed for perinatal death attributed to congenital malformations. In the group that died in the neonatal
period, the emergency $\mathrm{CD}$ rate was significantly lower where anomaly was detected versus undetected ( $17.5 \%$ versus $31 \%$ ). Further, in contrast to period, the emergency $C D$ rate was significantly lower where anomaly was detected versus undetected (17.5\% versus $31 \%)$. Further, in contrast to
undiagnosed anomalies, the indication for emergency $C D$ was more often maternal in the diagnosed group $(42 \%$ versus $19 \%$, $p=0.019)$. When a diagnosis of lethal congenital anomaly has been made in the prenatal period, the reduction in the emergency CD rate by almost half in this study supports a pivotal role for prenatal diagnosis in optimizing maternal care.
\end{abstract}

\section{Introduction}

Evidence based medicine has shown that vaginal delivery carries the least maternal morbidity when compared to caesarean delivery (CD) the majority of patients will deliver vaginally, if there is deterioration in the fetal condition, as demonstrated by abnormalities in a fetal

heart rate tracing, the mother will likely be delivered by emergency caesarean section. Caesarean delivery (CD), particularly when carried out in the emergency setting, is associated with greater morbidity for both mother and child.
blood loss, iatrogenic organ injury, haematoma and urinary tract infections. Additionally, emergency $C D$ carries up to 6 times the risk of the $\begin{array}{ll}\text { mother developing postnatal depression. } & \text { The most common indication for emergency CD is non-reassuring fetal testing as suggested by fetal } \\ \text { heart rate monitoring or fetal blood sampling. } & \text { Fetuses with a major congenital abnormality may be particularly prone to such intrapartum }\end{array}$ heart rate monitoring or fetal blood sampling. $\quad$ Fetuses with a major congenital abnormality may be particularly prone to such intrapartum fetal abnormality in advance of delivery has a meaningful impact on obstetric management, for example, in the avoidance of unnecessary caesarean delivery.

Methods

The three major Dublin maternity hospitals maintain detailed records of pregnancy and perinatal outcome. We evaluated a consecutive retrospective cohort of 211,163 patients, delivered of infants weighing $500 \mathrm{~g}$ or more in these three tertiary referral centers from January
1995 to December 2004 , for perinatal death attributed to congenital malformation. Two comparative cohorts were created, comprising prenatally diagnosed and prenatally undiagnosed lethal congenital anomalies. In the former group, a diagnosis of likely lethal fetal abnormality had been made prior to delivery and this information was available to clinical staff managing delivery. We excluded from the database all patients who received no antenatal care and cases in which perinatal death was attributed to a congenital abnormality not amenable to prenatal diagnosis. chorionic villus sampling, parity, gestational age at first antenatal visit, specific prenatal diagnosis, fetal ultrasoun dinformation was collected: gestational age at delivery, birth weight, whether labour was induced, mode of delivery (vaginal delivery, elective CD or emergency $\mathrm{CD}$ ), indication for emergency $\mathrm{CD}$, type of congenital abnormality, and final perinatal autopsy result. Continuous data were compared by independent sample t-test and categorical data by chi-square test.

\section{Results}

During the 10 year study period a total of 211,163 patients were delivered of infants weighing at least $500 \mathrm{~g}$. Perinatal death attributable to congenital malformation occurred in 692 pregnancies during the study period (incidence $0.33 \%$ ). The inclusion criteria for this study were met by 634 patients, amongst whom the fetal abnormality was correctly diagnosed prenatally in $62.1 \%$ (394/634). No significant differences were identified between he prenatally diagnosed and undiagnosed groups with respect to maternal age, parity, gestational age at delivery or infant birth weight. A total of 240 (37.9\%) patients were delivered of infants with lethal abnormalities which were not prenatally diagnosed and were heart rate abnormalities during labour.

In 237 (37.4\%) cases an intra-uterine death (IUD) was diagnosed before delivery of the fetus, and in the remaining $397(62.6 \%)$ cases the infant died during the neonatal period. The incidence of emergency $C D$ was found to be significantly lower in the cohort in which the congenital abnormality had been diagnosed prenatally. The rate of emergency $\mathrm{CD}$ in this prenatally diagnosed group was $17.5 \%$, compared with the prenatally diagnosed group $42 \%$ of $C D$ were due to a maternal cause (eg diabetes, pre-eclampsia, multiple previous $C D$, cephalopelvic disproportion), while in the prenatally undianosed group such maternal reasons for CD were only found in $19 \%$ of cases. Nonreassuring fetal testing, as evidenced by an abnormal fetal heart rate tracing or fetal scalp pH, was the most common cause for emergency $\mathrm{CD}$, and was reduced by over 2 fold when the congenital abnormality was diagnosed prenatally.

\section{Discussion}

In Ireland patients who wish to terminate a pregnancy must travel to another jurisdiction to do so, as pregnancy termination for fetal malformation is not legally available. Therefore, patients in Ireland are more likely to be faced with the scenario of a live fetus in utero labour, and if knowledge of the lethality of the malformation is not available, the patient will likely undergo emergency caesarean delivery. The objective of our study was to evaluate to what extent prenatal knowledge of a lethal fetal malformation was of benefit to patients, as it may result in the safe avoidance of an emergency caesarean delivery. While some consider the main reason to undergo prenatal diagn
fetal abnormalities is to provide patients with an opportunity for pregnancy termination, our objective was to evaluate whether prenatal diagnosis has other practical benefits for patients, other than a simple decision to continue or interrupt the pregnancy.

Our data clearly demonstrate that advanced knowledge of the condition of the fetus can have a significant impact on the level of intrapartum fetal monitoring required and consequently on mode of delivery of the affected fetus. In our cohort of 634 perinatal deaths attributable to congenital malformation, we have demonstrated the significant positive impact that prenatal diagnosis could have on mode of delivery. The reduction of emergency caesarean delivery by nearly $50 \%$ in this setting may be a key benefit of prenatal diagnosis, and has the potentia The consequent reduction in maternal operative morbidity and positive impact on subsequent pregnancy management should not be underestimated.

\section{Acknowledgements}

I would like thank all those in Rotunda for the help and inspiration they have been. I would also like to thank RCSI for helping with funding this project.

MA Dempsey ${ }^{1}$, FM Breathnach $\quad{ }^{1,2}$, M Geary $^{2}$, C Fitzpatrick $\quad{ }^{3}$, M Robson $\quad{ }^{4}$, FD Malone ${ }^{1,2}$

${ }_{2}$ Royal College of Surgeons in Ireland, Dublin

${ }_{3}^{2}$ Rotunda Hospital, Parnell Square, Dublin 1
${ }^{4}$ Coombe Women's Hospital, Dolphins Barn, Dublin 8
${ }_{\text {National Maternity Hospital, Holles St, Dublin } 2}$

Email: markydempsey@amail.com

\section{References}

1. Hall MH, Bewley S. Maternal morbidity and mode of delivery. Lancet 1999; 354: 776-777.

A retrospective study of intra-operative and postoperative maternal complications

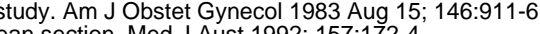

5. Rageth JC, Juzi C, Grossenbacher H. Delivery after previous caesarean section: A risk evaluation. Am J Obstet Gynecol 1999; 93:332-337.

6. Wareham V, Bain C, Cruickshank D. Caesarean section audit by Peer review. Euro J Obstet Gynecol reprod Biol 1993; 48:9-14. 